\title{
The Lassa Virus Epidemic in Mango, Togo: Therapeutic Route of the First 4 Cases
}

\author{
Majesté Ihou Wateba ${ }^{1 *}$, Aimé Dakévi¹, Lidawu Bawé1, Abago Balaka², Awereou Kotosso ${ }^{1}$ \\ ${ }^{1}$ Tropical and Infectious Diseases Service of CHU SO, Lomé, Togo \\ ${ }^{2}$ Internal Medicine Service CHU of SO, Lomé, Togo \\ Email: *majeste7@yahoo.fr
}

How to cite this paper: Wateba, M.I., Dakévi, A., Bawé, L., Balaka, A. and Kotosso, A. (2017) The Lassa Virus Epidemic in Mango, Togo: Therapeutic Route of the First 4 Cases. Advances in Infectious Diseases, 7, 135-142.

https://doi.org/10.4236/aid.2017.74014

Received: July 24, 2017

Accepted: November 14, 2017

Published: November 17, 2017

Copyright (c) 2017 by authors and Scientific Research Publishing Inc. This work is licensed under the Creative Commons Attribution International License (CC BY 4.0).

http://creativecommons.org/licenses/by/4.0/

\begin{abstract}
Introduction: Lassa viral hemorrhagic fever is caused by the Lassa virus. The aim of our study is to describe the therapeutic itinerary of the 4 cases of Lassa virus hemorrhagic of February-March 2017 epidemic that occurred in Mango. Methodology: Our study is a transverse retro-prospective and descriptive study from February, $1^{\text {st }}$ to March, $31^{\text {st }} 2017$ that dealt with 4 confirmed Lassa fever cases declared positive on the PCR basis; hospitalized or deceased at the hospital "Esperance" of Mango; support center of Lassa viral hemorrhagic fever. Results: we reported 4 clinical observations of Lassa viral hemorrhagic fever diagnosed on the PCR basis during the Lassa fever epidemic. Patients came from Benin ( 2 cases) or from Burkina-Faso (1 case) and were 25, 60, 52 years old and a premature baby of 13 days. The reasons for admission were external hemorrhage, a pultated tonsillitis and abdominal pains. Fever was observed for all the cases. Complications were marked by hemorrhages and shocks. Only two patients benefitted from Antiviral therapy with Ribavirin and were declared healed. The other two patients did not benefit from the treatment due to diagnostic and therapeutic delays. Lethality was $75 \%$ (3 cases) with a highly secured burial. Effective management of contacts was established. Conclusion: Diagnostic and therapeutic delays of patients are responsible of the Dark Prognosis of Lassa fever during the epidemic.
\end{abstract}

\section{Keywords}

Fever, Hemorrhage, Lassa Virus, Mango (Togo)

\section{Introduction}

Hemorrhagic fevers of viral origin (FHV) are by no means a phenomenon of recent discovery. They associate with a generally high fever hemorrhagic signs of 
varying intensity with an important lethality rate sometimes [1]. Lassa fever is a viral disease belonging to the FHV group occurring in epidemics, mainly in West Africa due to a virus of the Arenaviridae family: Lassa virus [2], whose first epidemic was documented in 1969 in the town of Lassa in the northeastern part of Nigeria, which led to the isolation of the Lassa virus. This disease is a real scourge in West Africa, where it is responsible for deadly epidemics, and it is estimated that the number of infections is 100,000 to 300,000 and that of the deaths about 5000 per year with a death rate of $1 \%$ to $15 \%$ [3] [4]. Pregnant women pay the heavy price because their death rate for Lassa fever reaches $30 \%$ and the fetus dies in $80 \%$ of cases [5]. Diagnostic confusion is common with the other FHV (Ebola, Marburg, dengue ...) and Lassa fever easily resembles the etiologies of tropical fevers (malaria, typhoid fever, septicemia...). Lassa fever constitutes a therapeutic emergency, imposing administration of Ribavirin within six days following the apparition of the first signs, in the hope to give better chances to the patient to be healed [5]. In Togo, the therapeutic itinerary of patients infected with Lassa virus remains poorly known due to the multiplicity of unqualified participants in therapeutic care. This study therefore aims to describe the therapeutic itinerary of the first 4 cases of FHV of Lassa virus during the February-March epidemic in Mango, Togo.

\section{Method}

This study was carried out simultaneously at the Mango Prefectural Hospital Center and at the United States hospital named Esperance hospital of Mango in Togo. It was a cross-sectional study with a descriptive aim from 1 February 2017 to 31 March 2017, which required our stay in the epidemic zone for a good completeness of information. Our study focused on the first 4 cases of Lassa fever hospitalized or dead in the hospital of Mango and the hospital of Esperance in which the diagnosis of Lassa fever was posed on the positivity of PCR. We therefore report 4 clinical cases of hemorrhagic fever with Lassa virus.

\section{Results}

\subsection{Clinical Case Number 1}

She were a 25-year-old woman, a housewife, a second gesture, a nulliparous (1 stillbirth) resident in Ouragaye (Burkina Faso), admitted to the Esperance hospital on 23 February 2017 at 11:00 54 minutes for externalized hemorrhages with fever.

\subsubsection{Itinerary of the Patient}

The beginning of the disease was the 15 February 2017, in fact 8 days before admission, marked by dizziness of sudden occurrence imposing decubitus on the pregnant with chills in a febrile context, which led to a self-medication based on Paracetamol with regression of fever. The pregnant woman consulted in a CMS in Ouragaye on 17 February where she was hospitalized and treatment based on 
Artemether and Paracetamol was instituted with a transient improvement in symptoms. She went out of the hospital on the 18th and ended up entering labor of childbirth followed by a miscarriage at home on February 19, 2017, complicated with immediate postpartum hemorrhage. She was then re-admitted to the same hospital in Ouragaye where she received a transfusion of six pockets of isogroup, isorhesus, globular pellets. Bleeding from the ears, mouth, nose and vagina appeared a few hours later after admission and her condition quickly became precarious. In view of the worsening of the clinical condition despite the transfusion, the family decided to consult the hospital of Esperance on 23 February for better care.

\subsubsection{Diagnosis and Treatment Process}

On admission the patient was in poor general condition with clinical examination:

Clinical infectious syndrome;

$>$ Bilateral hypoacousia;

$>$ Intense agitation and profuse sweating;

A stage III coma with a Glasgow score of $6 / 15(\mathrm{Y}=1, \mathrm{~V}=2, \mathrm{M}=3)$;

Hemorrhagic syndrome with externalized bleeding from the ears, mouth, nose, and vagina as well as the anus.

This clinical chart reflects a state of decompensated hypovolemic shock; Intensive car measures were undertaken and a full blood sample for PCR was withdrawn. The antiviral protocol with Ribavirin was not instituted because of the time lapses to put under Ribavirin. The patient was diagnosed with sepsis, nose, mouth, vagina, anus, with fever and active bleeding. The patient died on March 3, 2017 (8 days in isolation). The body was isolated for a secure burial and a case management was instituted.

\subsection{Clinical Case Number 2}

This was a 13-day-old preterm newborn from a poorly followed pregnancy; Residing with his parents in Papani in Benin living in insanitary conditions with the notion of regular consumption of rodents; Admitted to the hospital of the Esperance on February 24, 2017 for fever.

\subsubsection{Itinerary of the Patient}

The onset of symptoms was three days before admission to Papani, marked by the sudden apparition of hemoptysis with abundant hemodynamic (hemodynamic) repercussions in a febrile context in the mother of the newborn, 28 years second nulliparous (one stillborn); Hospitalized in Papani on 9 February. Forty-eight hours later, tonico-clonic convulsive seizures prompted a prophylactic caesarean section on 11 February. Four hours after this Caesarean section occurs the death of this new baby in a context of externalized bleeding (nostrils, ears, mouth, vagina) and hyperthermia. Papani's doctors placed the newborn, his father and his paternal grandmother in solitary confinement after sending a blood sample from 
the dead mother to the laboratory, which returned positive to the Lassa virus on February 14, 2017. However, on the night of February 13, the father escaped with the newborn and his grandmother from the hospital and returned to the village of Tangueta in Togo on 14 February 2017. The doctors at the CMS Papani immediately contacted the Esperance Hospital and the father, the newborn and his grandmother who became his nurse, were placed in solitary confinement at their home, where twice-daily monitoring based on temperature was initiated. It was during this surveillance that the newborn presented a fever which motivated his hospitalization on February 24, 2017.

\subsubsection{Diagnosis and Treatment Process}

On admission, the premature patient was in poor general condition with a pale and vague face, with clinical examination:

$>$ Clinical infectious syndrome;

Decompensated clinical anemic syndrome marked by:

In view of this highly suggestive clinical chart, the diagnosis of Lassa fever was made and intensive care measures were undertaken in the newborn and the protocol with Ribavirin initiated. An adjuvant treatment of: antibiotic, antimalarial, antipyretic was instituted and a blood sample for PCR was made. The evolution in isolation was unfavorable with the appearance of bloody vomiting on 08 March with external bleeding (nose, mouth, anus) leading to death on March 09, 2017 (12 days in isolation). The body was isolated for a secure burial and a case management was instituted.

\subsection{Clinical Case Number 3}

She was a 60 -year-old patient, case contact with clinical Case 2, a newborn babysitter after her mother's death, living in insalubrious conditions, regularly consuming rodents and living in Yaklè (a village located at $07 \mathrm{~km}$ from Village of Gando); Hospitalized and placed in isolation at the hospital of the Esperance since 24/2/2017 because it was case of contact of the clinical Case 2 .

\subsubsection{Clinical Evolution}

Five days after her admission on 01 March 2017: the grandmother reported spontaneous retro-sternal pain with odynophagia and hyperthermia.

\subsubsection{Diagnosis and Treatment Process}

The clinical examination made it possible to note:

$>$ Clinical infectious syndrome;

Exudative tonsillitis;

A left unilateral hypoacousia;

Good hemodynamic status.

In view of this highly suggestive clinical picture, the diagnosis of Lassa fever was made and the antiviral protocol with Ribavirin was initiated as well as an adjuvant treatment based on antibiotic, analgesic and antipyretic. A blood sam- 
ple was collected for PCR. The evolution in isolation was favorable:

> March 10: last dose of Ribavirin and control PCR control;

Darch 12, 2017: PCR negative control of the Lassa virus, signing the patient's cure with its execution on March 14, 2017. A weekly follow-up was instituted.

\subsection{Clinical Case Number 4}

This was a 52-year-old livestock farmer whose last trip to Benin was three weeks before the onset of symptoms; Residing in Gando (village located $50 \mathrm{~km}$ from the Nationale N.1 of Mango on the Togo-Benin border); Admitted to the CHP of Mango on 12 March 2017 for fever and abdominal pain.

\subsubsection{Itinerary of the Patient}

The onset of symptoms dated back ten days earlier, marked by the sudden beginning of intense pains in the type of stabbing, localized in the right lumbar fossa and secondarily generalized throughout the abdomen; Associated with severe asthenia, dizziness imposing decubitus on the patient; All in a febrile context. A first hospitalization of two in Gando on 03 March 2017 led to an antimalarial, antispasmodic and antipyretic treatment with a transient change in symptomatology. Four days later (07 March), diarrhea appeared at first, rapidly becoming uncontrollable, dizziness with hyperthermia at 40 degrees Celsius despite the use of Paracetamol with worsening asthenia, requiring the patient to re-visit the CMS Gando where he was hospitalized again and treated with the same medications as previously with the association this time of an antibiotic therapy without success. When the symptoms worsened, the patient escaped with the help of his companions on 12 March 2017 to consult with the CHP de Mango for better care.

\subsubsection{Diagnosis and Treatment Process}

At admission the patient was in poor general condition with clinical examination:

$>$ Clinical infectious syndrome;

$>$ Decompensated clinical anemic syndrome;

A stage II coma with a Glasgow score of $8 / 15(Y=3, V=3, M=2)$.

Resuscitation was initiated in the patient and an analgesic, antibiotic and antimalarial treatment was instituted. It should be noted that since the Mango hospital is not the center for the management of Lassa fever, the reflex has not been evoked by Lassa fever, but rather by classical diseases and faced with the failure of conventional therapies that the diagnosis of lass a fever was evoked. Thus, three diagnostic hypotheses were evoked:

Severe malaria anemic neurological form;

Digestive salmonellosis;

Lassa fever.

The evolution in hospitalization was unfavorable marked after two hours by the non-thermal defervescence which required the opinion of a doctor informing them of cases of Lassa in the zone. The patient was placed in isolation and a blood sample was collected for PCR. The death occurred 12 hours later in a con- 
text of hyperthermia and respiratory distress. A secure burial was carried out and case management was instituted. Twenty-four hours later, the result of PCR was positive for Lassa virus.

\section{Discussion}

\subsection{General Considerations}

Diagnostic delay was found in all cases except the newborn and grandmother who were identified as contacts of a dead subject. This delay constitutes the real handicap factor determining the prognosis of the disease since Ribavirin must be administered within the first 6 days. This is due to the multiplicity of unqualified interveners who mark the route of the cases, which is detrimental to cases and to the community because of the high contagiousness of the epidemic. Indeed, customary and funerary rites consisting of washing dead bodies by their close relatives are legion in Black Africa [6]. Moreover, during Ebola outbreak in Liberia some histories reveal that the parents were rinsing themselves with the water used to wash the deceased, with the aim of attracting his favors, which is responsible for the dissemination of the disease. While recent studies by the World Health Organization (WHO) reveal that there are 32 doctors per 10000 inhabitants in the European Region, in the city of Mango there are 20 doctors for 42397 inhabitants, which corresponds to a ratio of 05 doctors per 10000 inhabitants [7], which creates a vacuum that is quickly occupied by traditional practitioners close to the communities, where they are considered as leaders of opinion.

\subsection{Clinical Data}

In this study, all patients were febrile and the fever was constant. The work of Simpson and have reached the same conclusion, indicating a slow and insidious start making the diagnosis difficult at first, the fever appearing later [1]. Three out of four patients (75\%) had bleeding episodes in the present study, contrasting with WHO FHV studies, which showed that, despite the FHV name, the clinical picture of FHVs only involved hemorrhages In less than half of confirmed cases of Ebola infection and in less than $20 \%$ of confirmed cases of Lassa fever [5]. The high proportion of hemorrhagic episodes in our study could therefore be explained in part by the small size of the confirmed cases, but also and above all by the delay of these cases which leads them to the stage of complications in particular hemorrhagic complications in charge. However, it should be noted that in the neonate of clinical Case 2 in particular and children in general, the serious course could be due to the immaturity of their immune system; Which is ineffective against perinatal infections; How much more so in front of an FHV, especially that of Lassa! There is, therefore, the special malnutrition situation in this mother orphan due to insufficient nutritional intakes (artificial milks, breast feeding of the 60 -year-old grandmother) constituting a true factor of immunosuppression, aggravating the course of the disease. 


\subsection{Therapeutic Data}

The treatments received by patients seem justified and adapted to our realities and international recommendations [2]. The delay before admission is responsible for the 2 cases of death (clinical Cases 1 and 4 ) observed during this epidemic. However, in the newborn of clinical Case 2, who has not suffered any therapeutic delay, death can be elucidated by the following arguments:

Ribavirin has no proven efficacy in newborns and children [5], so there are very few studies available on this subject since in most cases, Lassa fever is responsible for more than $80 \%$ of miscarriages [5] as in clinical Case 1.

$>$ The immaturity of the child's immune system, especially which of the newborn, combined with the special malnutrition zone, is an important factor in immunosuppression, which is favorable to the severe progression of Lassa fever and this same treatment.

Finally, it should be noted that Ribavirin treatment has no side effects, especially anemia and alopecia, even in adults [5].

\subsection{In Relation to Isolation}

Psychological repercussions are very important, particularly in Africa where community life is the main characteristic. Patients quickly fall into depression, refuse to eat or even accept care. The patient is thus categorized in his community as a bird of ill omen, even after his recovery as the patient of the clinical Case 3 who, although cured, was always treated as contagious by the villagers who avoided contact with her. This state of affairs emphasizes more than all the importance and the preponderant place of psychotherapy in the management of this FHV.

\section{Conclusion}

Lassa fever is a viral hemorrhagic fever caused by the Lassa virus, the first outbreak of which has been documented in the village of Lassa. The positive diagnosis is mainly based on PCR and the culture of the virus in other heavens. The curative treatment is based on the administration of Ribavirin in the first six days after the onset of the first signs. However, it is clear that the consultation period is long in Africa where the therapeutic itinerary of the patients is marked by several unskilled participants; responsible of the delay of the diagnosis and increase the risk of death.

\section{References}

[1] Simpson, D. (1979) Fièvres hémorragiques virales de l'homme. [Viral Hemorrhagic Fevers of Man.] Bulletin of the World Health Organization, 57, 19-32.

[2] Saltzmann, S. (1978) La fièvre de Lassa. [Lassa Fever.] Edition of Missionary Groups, Strasbourg, 46.

[3] Gunther, S. and Lenz, O. (2004) Lassa Virus. Critical Reviews in Clinical Laboratory Sciences, 41, 339-390. https://doi.org/10.1080/10408360490497456 
[4] Bulletin of the Ministry of Health of Togo (2016) Surveillance of Haemorrhagic Viral Fevers and Contact Tracing, Togo, June. 22 p.

[5] WHO (2014) Clinical Management of Viral Haemorrhagic Fever Cases: A Guide for the Front-line Health Worker. WHO, 10-23.

[6] Decaillet, M. (2015) Ebola: face à la rumeur. [Ebola: Facing the Rumor.] Lille, 16 p.

[7] WHO (2009) World Health Statistics. WHO, 95-109. 